Conclusion This study indicated that Ca level in breast milk might be associated with the underweight at 6 months old in rural Bangladesh.

\section{P2-389 USE OF HEALTHCARE IN A SOCIAL NETWORK OF MEN WHO HAVE SEX WITH MEN IN BRAZIL}

doi:10.1136/jech.2011.142976l.19

${ }^{1} S$ Brignol, ${ }^{1}$ I Dourado, ${ }^{*}{ }^{2} \mathrm{M}$ Mello, ${ }^{2}$ A Pinho, ${ }^{3}$ L Kerr. ${ }^{1}$ Public Health Institute, Federal University of Bahia, Salvador, Bahia, Brazil, ${ }^{2}$ Fiocruz Foundation, Ministry of Health, Rio de Janeiro, Rio de Janeiro, Brazil; ${ }^{3}$ Medical School, Federal University of Ceara, Fortaleza, Ceara, Brazil

Introduction It is known that men access health services less frequently than women. According to WHO it is even less frequent among men who have sex with men (MSM) which increases the vulnerability of this group to HIV/AIDS and other STD.

Methods To describe the use of healthcare on HIV/STD among MSM, data were collected using respondent driven sampling as part of the baseline of the Brazilian behavioural and serologic surveillance survey of 3859 MSM in 10 cities in 2009. The analyses, conducted with 383 MSM from a major capital city-Salvador, Northeast Brazil, used a complex network theory, specifically two-mode networks with bipartite graph, classic statistics analysis of networks and projection. Results Among the participants, $57 \%$ referred to have access to general health services, with $55 \%$ of them having had at least one medical appointment within the past 2 years. Over half (56\%) did not know where to go for an HIV test; and $44 \%$ had an HIV test in the past year. Among those, $23 \%$ tested in a public health clinic, and $64 \%$ were given free condoms on the same facilities.

Conclusion To improve access to healthcare and to HIV testing among MSM is crucial to tackle the epidemic in Brazil, especially with the high HIV prevalence (12.6\%) reported in the country among this population group.

\section{P2-390 CARDIOVASCULAR AND DIABETES RISK IN PERSONS WITH PREDIABETES}

doi:10.1136/jech.2011.142976l.20

T Dzebisashvili. ${ }^{*}$ Moscow Regional Research Clinical Institute, Moscow, Russia

Aim To assess RR of type 2 diabetes (T2DM), overall and acute cardiovascular mortality and cardiovascular events in persons with impaired fasting glucose (IFG) and impaired glucose tolerance (IGT). Materials and Methods According to population based study among 2508 adults, the 3 -year risk of T2DM, overall and acute cardiovascular mortality and cardiovascular events (fatal and nonfatal myocardial infarction and stroke, coronary heart disease) was estimated in people with glucose metabolism abnormalities (GMA): IFG, IGT, IFG+IGT diagnosed in 2006 in comparison with normal glucose tolerance. RR and regression coefficient (B) was calculated using Coxregression analysis. RR of T2DM, cardiovascular events was adjusted for age, sex, BMI, systolic blood pressure (SBP), smoking.

Results Highest adjusted RR of T2DM were in IFG+IGT (11.2 [3.93-31.65], $\mathrm{p}<0.01)$. Lowest RR of T2DM were in isolated IGT (3.92 [1.11-13.90], $p=0.034)$. Adjusted $R R$ of cardiovascular mortality was significantly 3.2-fold higher in IFG. IGT and newly diagnosed T2D had significantly 3.6-fold and 2.3-fold greater risk of overall mortality. RR of cardiovascular events was significantly increased 2.2-fold in IFG and 2.7-fold in T2D. There was not linear association between blood glucose levels and cardiovascular mortality risk ( $p=0.095)$ in contrast to the continuous linear relationship observed between blood glucose levels and coronary heart disease risk $B=0.273(p=0.001)$.
Conclusion 3 year risk of T2DM is not equal at different early GMA: highest one - in IFG + IGT, lowest - in isolated IGT. IFG increased 3year risk of acute cardiovascular mortality. There was not linear association between blood glucose levels and cardiovascular mortality risk.

\section{P2-391 HBA1C FOR DIABETES MELLITUS DIAGNOSIS}

doi:10.1136/jech.2011.142976l.21

T Dzebisashvili." Moscow Regional Research Clinical Institute named, Moscow, Russia

Aims To evaluate diagnostic strategy with OGTT and/or HbA1c for lowing number of people with undiagnosed T2DM.

Materiales and Methods Population-based screening for glucose metabolism impairments (GMI) among 661 adults in Moscow Country was conducted in 2009. HbA1c was determined in 39 subjects with GMI.

Results Based on OGTT and HbA1c, 6 and 11 people had T2DM; 33 and 23 people had prediabets. Mean HbA1c (SD) was 7.9 (2.8) for T2DM, 6.0 (0.5) for IGT and 5.8 (0.7) for IFG and 6.5 (0.5) for IGT +IFG. The sensitivity/specificity $(\mathrm{Sn} / \mathrm{Sp})$ of $\mathrm{HbA1c}>6.5$ for T2DM were $66 \% / 78 \%$, Sn/Sp of HbA1c $>5.7-6.4 \%$ for IGT were $68 \% / 64 \%$, for IFG were $50 \% / 42 \%$ and for IGT + IFG were $50 \% / 42 \%$. Using Roc curve analysis, the single optimal HbA1c cut-point for detecting T2DM was $>6.0 \%$, (Sn/Sp: $50 \% / 100 \%$ ), for IFG was $<5.0 \%$ (Sn/Sp: $50 \% / 100 \%$ ) in normal weight (BMI 18-25) individuals. RR of T2DM was 7 (1.18-42.9) with HbA1c values 6.0-6.4\% and $>6.5 \%$, than those with $<6.0$ in normal weight individuals. $33.0 \%$ of undiagnosed T2DM had HbA1c levels $<6.5 \%$ (95\% CI $0 \%$ to $71 \%$ ) and $17 \%$ of people with T2DM had HbA1c levels $<6.0 \%$.

Conclusion OGTT and $\mathrm{HbA1c}$ are both relevant diagnostic criteria for dysglycemia as they correlate with the risk for developing T2DM. Choosing the HbA1c strategy rather than the OGTT strategy leads to diagnose more diabetes, although the consistency of both diagnostic criteria is low. The optimal HbA1c cut-point to detect T2DM was lower than $\mathrm{HbA1c}$ of $6.5 \%$ in normal weight individuals.

\section{P2-392 RISK FACTORS FOR DIABETES AND PREDIABETES}

doi:10.1136/jech.2011.142976l.22

T Dzebisashvili. ${ }^{*}$ Moscow regional research clinical institute of M.F.Vladimirsky, Moscow, Russia

Aim To estimate risk factors for developing type 2 diabetes (T2D), impaired glucose tolerance (IGT), impaired fasting glucose (IFG) and combination IGT + IFG

Material and Methods A population-based screening for glucose metabolism impairments using standard 2-h OGTT among 2508 adults in Moscow County was conducted. BMI, waist circumference (WC), systolic and diastolic blood pressure were estimated. Lipids (total, LDL, fasting triglycerides (TG)) measurements were performed among 661 persons. Also participants fill forms about other risk factors. RR of T2D, IFG, IGT and unstandardised regression coefficient (B) was calculated using Cox-regression analysis SPSS V.13.0 (adjusted for BMI and age). RR was considered significant if confidential interval was not included 1 and $p \leq 0.05$.

Results Risk factors for T2DM and prediabetes was age, BMI, systolic blood pressure. Also risk factors for T2DM was ischaemic heart disease, family history of diabetes, alcohol consumption, diastolic blood pressure (DBP), waist circumference (WS), raised triglycerides, reduced smoking $(B=-0.374, p=0.032)$. Risk factors for IGT was DBP, oral contraceptives. Risk factors only for IFG was physical inactivity, gender. Risk factors for IGT+IFG was budget, DBP, WS in men. 